\title{
Treatment in a ward for elderly patients with dementia in Japan
}

\author{
This article was published in the following Dove Press journal: \\ Neuropsychiatric Disease and Treatment \\ 5 March 2013 \\ Number of times this article has been viewed
}

\author{
Shogo Taniguchi' \\ Jin Narumoto' \\ Keisuke Shibata' \\ Nobutaka Ayani' \\ Teruyuki Matsuoka' \\ Aiko Okamura' \\ Kaeko Nakamura' \\ Hiroshi Shimizu \\ Kenji Fukui' \\ 'Department of Psychiatry, Graduate \\ School of Medical Science, Kyoto \\ Prefectural University of Medicine, \\ Kyoto, Japan; ${ }^{2}$ Umibeno-mori Hospital, \\ Kochi, Japan
}

Background: Japan has become the world's most aged country. The percentage of elderly people in Japan is estimated to reach $25.2 \%$ in 2013 , and the number of patients with dementia is estimated to reach 2.5 million in 2015. In addition to its deterioration of physical function and activities of daily living (ADL), behavioral and psychological symptoms of dementia (BPSD) often become major clinical problems, greatly annoying patients and their caregivers. In Japan, we utilize wards for elderly patients with dementia (WEDs) for BPSD treatment. However, there are few studies investigating the effectiveness of treatment in a WED. In such treatment, physical complications are a challenge physicians must overcome while treating BPSD and safely returning patients home or to the institutions in which they live. Therefore, we investigated the effectiveness of treatment in a WED, focusing on physical complications.

Methods: The subjects were 88 patients who were admitted to and discharged from a WED. Severity of dementia, basic ADL, and BPSD were investigated using the Clinical Dementia Rating, Physical Self-Maintenance Scale (PSMS), and Neuropsychiatric Inventory. Differences in characteristics between patients discharged from the WED because of physical complications and all other patients were also examined.

Results: We found significant improvements in the PSMS score and decreases in delusions and sleep disturbances in all patients. Patients discharged from the WED because of physical complications had significantly greater severity of dementia at discharge compared to all other patients.

Conclusion: Treatment in a WED seems to be effective for BPSD and ADL, but care should be taken regarding physical complications, especially in patients with advanced dementia.

Keywords: aged country, behavioral and psychological symptoms of dementia, physical complications, activities of daily living

\section{Introduction}

According to the White Paper on the Aging Society, Japan became the world's most aged country in 2005, overtaking Italy in terms of the rate of elderly people, and is expected to enter an era of an "unprecedented" aging society in the future. ${ }^{1}$ The estimated rates of elderly people are $31.6 \%$ for 2030 and $39.9 \%$ for $2060 .^{2}$ The prevalence of dementia has increased as people are aging, ${ }^{3,4}$ and patients with dementia are projected to number 2.5 million in 2015 and 3.2 million in $2025.5^{5}$ During the course of illness, behavioral and psychological symptoms of dementia (BPSD) often become major clinical problems for patients and their caregivers, and sometimes lead to hospitalization. To treat BPSD, special wards for elderly patients with dementia (WEDs) were introduced in Japan in 1988. The staff in these wards constitute a multidisciplinary team comprising
Correspondence: Shogo Taniguch Department of Psychiatry, Graduate School of Medical Science, Kyoto Prefectural University of Medicine, 465 Kajii-cho, Kawaramachi-Hirokoji, Kamigyo-ku, Kyoto 602-8566, Japan

Tel $+8 I 7525 I 5612$

Fax +81752515839

Emailshogo@koto.kpu-m.ac.jp 
a psychiatrist, experienced nurses, an occupational therapist (OT), and a psychiatric social worker (PSW) or psychologist. Each professional evaluates the patients and provides treatment based on their specialty, such as medication, care and nursing, coordination of social circumstances, recreation, and group psychotherapy.

Several studies have shown the effectiveness of BPSD treatment at special acute units for people with dementia in Western countries. ${ }^{6,7}$ In contrast, few studies have examined the course of BPSD during a stay in a WED in Japan. Therefore, we conducted a retrospective analysis of patients who were admitted to a WED, with a focus on physical complications, because these often interrupt treatment and lead to transfer to another hospital. ${ }^{8}$ Ukai et $\mathrm{al}^{9}$ and Ukai and Mizuno ${ }^{10}$ found a high frequency of physical complications in a WED that required treatment by specialists of other medical departments, and Ono et $\mathrm{al}^{11}$ reported that physical complication was a predictor of a longer stay in a WED. Therefore, to identify risk factors related to physical complications, we compared the characteristics of patients transferred to another hospital or ward because of physical complications with those of all other patients. In addition, we investigated the effect of the use of antipsychotics on dementia severity, basic activities of daily living, and BPSD.

\section{Methods}

\section{Subjects and procedure}

We performed a retrospective chart review of 88 patients with dementia who were admitted to the WED at Umibeno-mori Hospital, Kochi, Japan between June 1, 2008 and May 31, 2009 and were discharged by January 1, 2010. The WED staff consists of a full-time psychiatrist, experienced nurses, a full-time OT, and a full-time PSW. In the WED, treatment includes medication and education about dementia and BPSD provided by the psychiatrist, care and nursing by the experienced nurses, preparation of social support by the PSW, and rehabilitation by the OT. The Umibeno-mori Hospital WED provides patients a familiar environment in addition to medication. For example, there are kitchens and a living room with televisions and sofas. Tansu (Japanese traditional cabinets) and tatami (Japanese traditional mats) are in patients' private rooms, and staff members put on casual clothing so as to provide patients a homelike atmosphere in an attempt to moderate their anxiety, strain, and wariness. Moreover, patients are provided with recreational activities such as hiking and tea groups so that they can have an enjoyable time and keep a regular daily rhythm, to prevent disuse syndrome, and to moderate BPSD. We provide group psychotherapy such as life reviews to stimulate their cognitive functions. Above all, since there are only elderly patients in the WED, conversation and communication happen naturally between them. All patients were admitted from home, an institution, or other wards at Umibeno-mori Hospital or other hospitals and were discharged to home, an institution, or other wards or hospitals.

We made the final diagnosis of dementia at discharge based on the text version of the fourth edition of Diagnostic and Statistical Manual of Mental Disorders (DSM-IV-TR). ${ }^{12}$ Standardized clinical diagnostic criteria were used to characterize the type of dementia. Diagnoses of Alzheimer's disease (AD), vascular dementia, dementia with Lewy bodies (DLB), and frontotemporal dementia (FTD) were made in accordance with NINCDS-ADRDA, ${ }^{13}$ NINDS-AIREN, ${ }^{14}$ and DLB consensus criteria described by McKeith et al, ${ }^{15}$ and FTD consensus criteria described by Neary et al, ${ }^{16}$ respectively.

Demographic and clinical data were collected from charts. The severity of dementia was evaluated using the Clinical Dementia Rating (CDR), ${ }^{17}$ basic ADL was determined using the Physical Self-Maintenance Scale (PSMS), ${ }^{18}$ and the presence of BPSD was established using the Neuropsychiatric Inventory (NPI) ${ }^{19}$ at admission and discharge. The CDR is an observation scale for the assessment of six domains memory, orientation, judgment, community affairs, home and hobbies, and personal care - with scoring as follows: CDR 0, healthy; CDR 0.5, questionable dementia; CDR 1, mild dementia; CDR 2, moderate dementia; and CDR 3, severe dementia. The PSMS consists of six items on the performance of the physical activities of using the toilet, self-feeding, selfdressing, self-grooming, self-ambulating, and self-bathing, with a lower total score indicating more ADL impairment. The NPI is a clinical instrument with which the caregiver evaluates the patient's 12 domains of neuropsychiatric symptoms in dementia: delusions, hallucinations, agitation, depression, anxiety, euphoria, apathy, disinhibition, irritability, aberrant motor behavior, sleep disturbances, and eating problems.

All chart reviews were performed by one rater (ST). The interrater reliability was established through independent rating by a skilled rater (NA) of 10 randomly sampled subjects. The interrater intraclass correlation coefficients were 0.96 and 0.96 for the CDR and 0.80 and 0.76 for the PSMS at admission and discharge, respectively, and the kappa statistics for each NPI subscale were 0.8-1.0. This study was approved by the Umibeno-mori Hospital ethical committee and was performed in compliance with the principles of the Declaration of Helsinki. Patient records were used carefully and anonymously. 


\section{Statistical analysis}

To investigate the effectiveness of treatment in the WED, we compared the scores for the CDR and PSMS and the presence of each NPI subscale at admission with those at discharge via a Wilcoxon signed rank test and a $\chi^{2}$ test. We also compared the characteristics of patients transferred to other wards or other hospitals because of physical complications with those of all other patients by using a Mann-Whitney $U$-test for continuous variables and a $\chi^{2}$ test for categorical data. In addition, we compared the CDR and PSMS scores and the number of patients with BPSD both at admission and discharge between patients who were prescribed antipsychotics and those who were not prescribed antipsychotics by using a Mann-Whitney $U$-test for continuous variables and a $\chi^{2}$ test for categorical data. Data were analyzed using SPSS (v12.0 J) for Windows (IBM Corporation, Armonk, NY, USA). A $P$-value $<0.05$ was considered significant. A Bonferroni correction was used to correct for multiple comparisons.

\section{Results}

The characteristics of the 88 patients ( 44 males, 44 females) are shown in Table 1. AD was the most common diagnosis (AD, 61\%; vascular dementia, 18\%; DLB, 5\%; FTD, 1\%; others, $17 \%$ ). Psychotropic prescriptions did not differ significantly at admission and discharge. About half of the patients were prescribed antipsychotics at admission. The number of patients with some physical complications increased slightly at discharge. At admission, there were no patients with severe physical complications requiring treatment at other wards or hospitals, but about one-third of patients had severe physical complications at discharge.

The PSMS score at discharge was significantly higher than that at admission, whereas the CDR score did not change significantly (Table 2). Regarding the presence of each NPI item (Figure 1), about $33 \%$ of patients had agitation, aberrant motor behavior, and sleep disturbances at admission. At discharge, there were large decreases in the percentages of patients with delusions and sleep disturbances and decreases in other NPI items, except for depression, euphoria, and irritability.

Table 3 shows a comparison of the characteristics of 29 patients transferred to other wards or hospitals because of physical complications and 59 patients discharged without severe physical complications from the WED to home, an institution, or another ward or hospital. There was a significant difference in the CDR score at discharge between these groups, but not for other characteristics, including the use of antipsychotics.
Table I Clinical characteristics of all patients at admission and at discharge from the WED

\begin{tabular}{|c|c|c|}
\hline Characteristics $(n=88)$ & At admission & At discharge \\
\hline Mean age (SD) & $81.3(7.08)$ & \\
\hline Gender (male/female) & $44 / 44$ & \\
\hline \multicolumn{3}{|l|}{ Diagnosis } \\
\hline$A D$ & & 54 \\
\hline VD & & 16 \\
\hline DLB & & 4 \\
\hline FTD & & 1 \\
\hline Others & & 13 \\
\hline \multicolumn{3}{|l|}{ Residence } \\
\hline Home & 33 & 18 \\
\hline Institution & 17 & 26 \\
\hline Other hospital & 18 & 22 \\
\hline Other ward & 20 & 22 \\
\hline Mean length of stay, days (SD) & & $70.9(70.7)$ \\
\hline Median length of stay, days & & 49 \\
\hline \multicolumn{3}{|l|}{ Psychotropic prescriptions } \\
\hline Antipsychotics & 47 & 48 \\
\hline Risperidone & 11 & 12 \\
\hline Olanzapine & 3 & 3 \\
\hline Quetiapine & 16 & 16 \\
\hline Perospirone & 10 & 10 \\
\hline Aripiprazole & 1 & 1 \\
\hline Typical antipsychotics & 11 & 11 \\
\hline Benzodiazepine & 12 & 11 \\
\hline Antidepressants & 18 & 15 \\
\hline Mood stabilizers & 20 & 23 \\
\hline Acetylcholinesterase inhibitors & 27 & 22 \\
\hline Physical complications & 69 & 75 \\
\hline Severe physical complications & 0 & 29 \\
\hline Pneumonia & 0 & 8 \\
\hline Fracture & 0 & 3 \\
\hline Urinary infection & 0 & 3 \\
\hline Chronic subdural hematoma & 0 & 2 \\
\hline Others & 0 & 13 \\
\hline
\end{tabular}

Abbreviations: AD, Alzheimer's disease; DLB, dementia with Lewy bodies; FTD, frontotemporal dementia; SD, standard deviation; VD, vascular dementia; WED, ward for elderly patients with dementia.

Table 4 shows the comparison of the CDR, PSMS scores, and the number of patients with BPSD between patients who were prescribed antipsychotics and those who were not prescribed antipsychotics at admission and at discharge. There were no significant differences; however, the CDR scores of patients who were prescribed antipsychotics tended

Table 2 CDR and PSMS scores (mean \pm SD) at admission to and at discharge from the WED

\begin{tabular}{llll}
\hline & At admission & At discharge & P-value \\
\hline CDR score & $2.34 \pm 0.78$ & $2.27 \pm 0.82$ & NS \\
PSMS score & $1.07 \pm 1.10$ & $1.33 \pm 1.22$ & $0.002 *$ \\
\hline
\end{tabular}

Notes: Wilcoxon signed rank test; *Bonferroni-corrected $P<0.01$.

Abbreviations: CDR, clinical dementia rating; NS, not significant; PSMS, physical self-maintenance scale; WED, ward for elderly patients with dementia. 


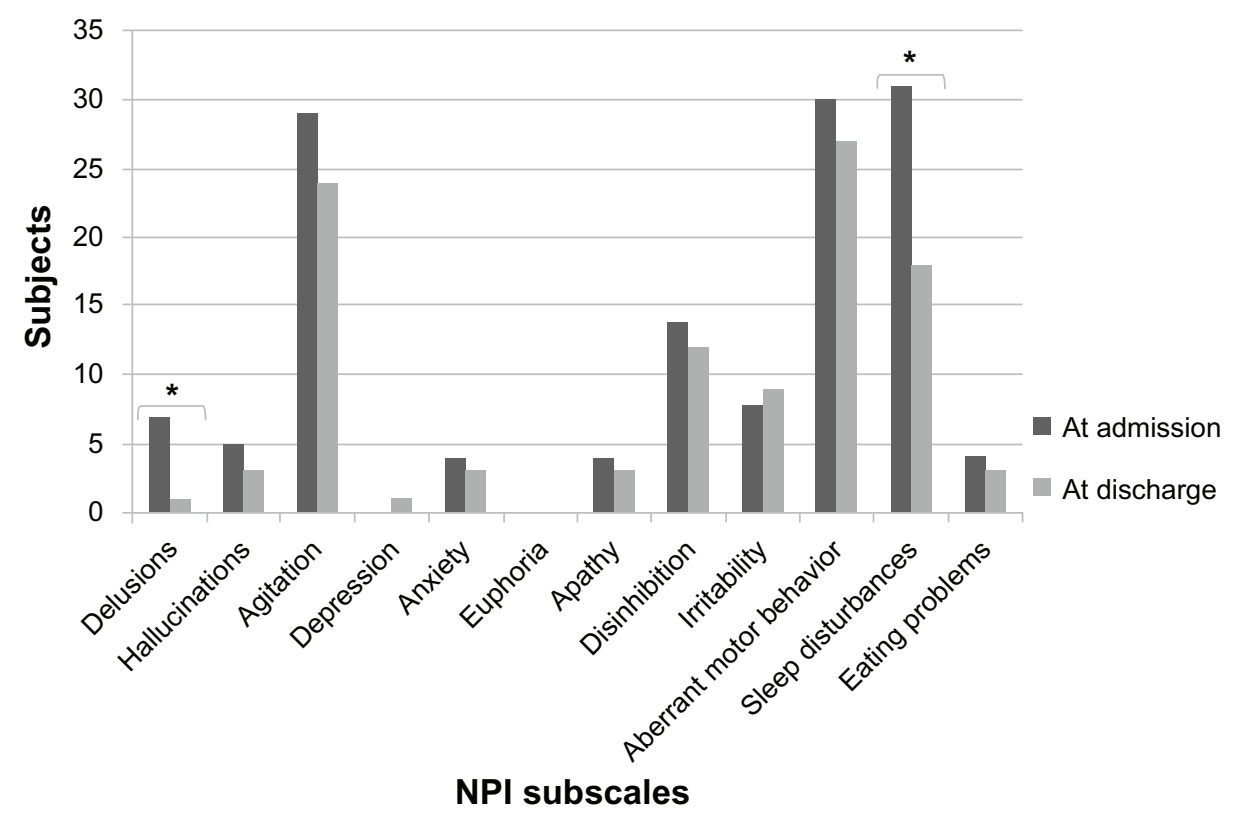

Figure I NPI subscale rates in 88 patients with dementia at admission to the ward for elderly patients with dementia and at discharge from same.

Notes: Almost all NPI subscale rates had decreased at discharge, especially for delusions and sleep disturbances. $*$ Uncorrected $P<0.05$, Bonferroni-corrected $P>0.05$.

Abbreviation: NPI, Neuropsychiatric Inventory.

to be worse than those of patients who were not prescribed antipsychotics at discharge.

\section{Discussion}

In this study, we used a retrospective chart review to investigate the utility of treatment in a WED and the characteristics of patients related to physical complications. The WED treatment significantly improved basic ADL and tended to decrease most BPSD, especially delusions and sleep disturbances. Patients transferred to other wards or hospitals because of physical complications had higher CDR scores at discharge. These results show the value of treatment in a
WED and also indicate a relationship between the severity of dementia and the occurrence of physical complications.

The improvement of BPSD through treatment of elderly people with dementia in special care units has been shown in previous studies, in which Bellelli et $\mathrm{al}^{6}$ found an overall reduction in behavioral disturbances and Colombo et $\mathrm{al}^{7}$ showed significant improvement of each element of BPSD. Our results are consistent with these studies and suggest that the treatment provided in a WED in Japan is effective in ameliorating BPSD. In regard to the reduction effect for delusions and sleep disturbances, we first considered the direct effects of medications. Second, as we explained in the

Table 3 Comparison of characteristics between patients discharged from the WED because of severe physical complications and those discharged without severe physical complications

\begin{tabular}{lll}
\hline & $\begin{array}{l}\text { Discharge without severe } \\
\text { physical complications }\end{array}$ & $\begin{array}{l}\text { Pischarge with severe } \\
\text { physical complications }\end{array}$ \\
\hline Number & 59 & 29 \\
Mean age at admission (years) & $81.6 \pm 6.0$ & $81.2 \pm 8.2$ \\
CDR at admission & $2.41 \pm 0.79$ & $2.62 \pm 0.56$ \\
CDR at discharge & $2.03 \pm 2.03$ & $2.66 \pm 0.61$ \\
PSMS at admission & $1.07 \pm 1.08$ & $0.76 \pm 0.58$ \\
PSMS at discharge & $1.62 \pm 1.35$ & $0.83 \pm 0.83$ \\
Physical complications & $46 / 13$ & $23 / 6$ \\
at admission (yes/no) & & $0.002 *$ \\
Prescription of antipsychotics & $34 / 25$ & NS \\
at discharge (yes/no) & & $14 / 15$ \\
\hline
\end{tabular}

Notes: Mann-Whitney U-test; Chi-square distribution; *Bonferroni-corrected $P<0.05$; **uncorrected $P<0.05$, Bonferroni-corrected $P>0.05$.

Abbreviations: CDR, clinical dementia rating; NS, not significant; PSMS, physical self-maintenance scale; WED, ward for elderly patients with dementia. 
Table 4 Comparison of the CDR and PSMS scores (mean \pm SD) and number of patients with BPSD between patients who were prescribed antipsychotics and those who were not prescribed antipsychotics at admission and at discharge

\begin{tabular}{|c|c|c|c|}
\hline & $\begin{array}{l}\text { Patients who were } \\
\text { prescribed antipsychotics }\end{array}$ & $\begin{array}{l}\text { Patients who were not } \\
\text { prescribed antipsychotics }\end{array}$ & $P$-value \\
\hline Number at admission & 47 & 41 & \\
\hline CDR score at admission & $2.4 \pm 0.74$ & $2.1 \pm 0.86$ & NS \\
\hline PSMS score at admission & $0.95 \pm 0.97$ & $1.1 \pm 1.2$ & NS \\
\hline Number of patients with & 44 & 39 & NS \\
\hline \multicolumn{4}{|l|}{ BPSD at admission } \\
\hline Number at discharge & 48 & 40 & \\
\hline CDR score at discharge & $2.4 \pm 0.86$ & $2.0 \pm 0.73$ & $0.04 *$ \\
\hline PSMS score at discharge & $\mathrm{I} . \mathrm{I} \pm \mathrm{I} .2$ & $1.6 \pm 1.2$ & NS \\
\hline Number of patients with & 36 & 27 & NS \\
\hline BPSD at discharge & & & \\
\hline
\end{tabular}

Notes: Mann-Whitney U-test; Chi-square distribution; *uncorrected $P<0.05$, Bonferroni-corrected $P>0.05$.

Abbreviations: BPSD, behavioral and psychological symptoms of dementia; CDR, clinical dementia rating; NS, not significant; PSMS, physical self-maintenance scale.

Methods section, supportive behavior by experienced staff members in the WED and familiar environments moderated their anxiety, strain, and wariness. Moreover, recreation, conversation, and communication between elderly patients contributed to stabilizing their mental states and regular daily rhythms. Hence, medications and the treatment environment in the WED might be effective for BPSD, especially relative to delusions and sleep disturbances. Regarding admission, Soto et $\mathrm{al}^{20}$ reported that the main cause of admission to a special acute care unit for elderly people with dementia was aggressiveness and that the frequencies of wandering and sleep disorders were high. Ono et $\mathrm{al}^{21}$ found that the major causes of admission to the WED were violence and wandering in men and wandering and care distress in women. In this study, the rates of agitation, aberrant motor behavior, and sleep disturbances were higher than those for other NPI items at admission, and these symptoms had decreased at discharge. This finding suggests that treatment in a WED addresses the symptoms that are mainly responsible for admission to the WED. However, many symptoms were still present at discharge, which indicates the importance of reducing BPSD further and supporting caregivers for coping with patients with dementia and BPSD.

Regarding the treatment itself, about half of the patients were prescribed antipsychotics both at admission and discharge. This suggests the persistent use of antipsychotics for BPSD in Japan, even after reports of an increased risk of mortality with the use of antipsychotics in people with dementia. ${ }^{22,23}$ The rate of use of antipsychotics did not differ significantly between patients transferred to other wards or hospitals because of physical complications and other patients; however, the CDR scores of patients who were prescribed antipsychotics tended to be worse than those of patients who were not prescribed antipsychotics at discharge. We consider it is possible that side effects of antipsychotics, such as anticholinergic and sedative effects, cause cognitive impairment in patients with dementia; we should thus prescribe antipsychotics carefully and make an effort to reduce the number of antipsychotic prescriptions we write. In contrast, we found a significant improvement in ADL. Thus, the multidisciplinary approach to the maintenance of ADL during the WED stay, including rehabilitation conducted by an OT, appears to be effective and may even improve ADL. Relatively shorter lengths of hospitalization may also have contributed to this result, since Yoshie et al found improved ADL levels for patients hospitalized for less than 1 year. ${ }^{24}$ Collectively, our results indicate that treatment in a WED is effective for ameliorating BPSD without worsening ADL.

About one-third of the patients had severe physical complications that led to referral to other wards or hospitals. The prevalence of severe physical complications is comparable to that in a previous report. ${ }^{25}$ Patients discharged because of physical complications had more impaired cognitive disorders than other patients. Physical complications have often been shown to be a common and serious problem in elderly patients with dementia. For example, Mitchell et al reported that pneumonia, febrile episodes, and eating problems were frequent complications in patients with advanced dementia and were associated with high 6-month mortality rates. ${ }^{26} \mathrm{We}$ should interpret the results in this study carefully, because it is possible that physical complications themselves cause cognitive impairment to patients with dementia. However, we believe that this study adds some knowledge about the relationship between physical complications and dementia severity, and we suggest that care should be taken regarding 
physical complications, especially in patients with advanced dementia.

The mean and median lengths of hospitalization in this study were 71 and 49 days, respectively, which are shorter than those in other WED studies. Kitamura et $\mathrm{al}^{27}$ reported median stays of 87 days for men and 74 days for women; Mizuno $^{28}$ found a rate of discharge within 3 months of $30 \%-40 \%$; Yoshie and Shiraishi ${ }^{23}$ reported mean and median stays of 353.4 days and 129.6 days, respectively; Ono et al $^{11}$ reported a median stay of 103 days; and Matsubara ${ }^{29}$ found that almost $85 \%$ of patients in wards specifically used for dementia treatment stayed for more than 91 days. The short length of hospitalization in this study might be due to the close relationships between the hospital and nearby institutions, which allow the relocation of patients to these institutions after BPSD improvement. Thus, social work is important to maintaining the function of a WED as an acute treatment unit for BPSD.

The results of this study must be interpreted with caution according to several limitations. First, it might be difficult to generalize the results of this study because there might be a slight difference between treatment in our hospital and in other hospitals. However, the aim and standard treatment in the WED is basically common in Japan based on regulation of WEDs, and we provided general treatment in the WED at Umibeno-mori Hospital. Second, the study was conducted retrospectively, and data obtained from chart reviews, physicians' estimations, and nurses' reports might be inaccurate. Third, the sample size was small. Fourth, the relationship between the severity of dementia and the occurrence of physical complications is controversial in this study, as described above. Fifth, different characteristics of patients with dementia (eg, ADL and BPSD) and their diagnosis might confound the treatment's effectiveness. Furthermore, we evaluated only the presence of BPSD and did not consider BPSD severity.

\section{Conclusion}

The present results suggest that treatment in a WED improved the ADL of patients with dementia and BPSD, particularly in terms of delusions and sleep disturbances. Patients who were discharged from the WED for treatment of severe physical complications had more impaired cognitive disorders compared to those who were discharged without severe physical complications. Since there have been few studies investigating the state and effectiveness of WEDs in Japan, we believe that this study adds some knowledge about the treatment provided by WEDs. Multicenter prospective studies are necessary to examine the effectiveness of treatment in a WED in a large number of patients with dementia whose characteristics are matched at baseline.

\section{Disclosure}

The authors report no conflicts of interest in this work.

\section{References}

1. Ministry of Health, Labour and Welfare. White Paper on the Aging Society (Summary) FY 2007. Tokyo: Ministry of Health, Labour and Welfare; 2007. Available from: http://www8.cao.go.jp/kourei/english/ annualreport/2007/2007.pdf. Accessed August 3, 2012.

2. National Institute of Population and Social Security Research. Population Projections for Japan (January 2012): 2011-2060. Tokyo: National Institute of Population and Social Security Research; 2012. Available from: http://www.ipss.go.jp/site-ad/index_english/esuikei/ gh2401e.asp. Accessed August 3, 2012.

3. Ferri CP, Prince M, Brayne C, et al. Global prevalence of dementia: a Delphi consensus study. Lancet. 2005;366:2112-2117.

4. Kawas C, Gray S, Brookmeyer R, Fozard J, Zonderman A. Age-specific incidence rates of Alzheimer's disease: the Baltimore Longitudinal Study of Aging. Neurology. 2000;54:2072-2077.

5. Nursing-Care for the Elderly Study Group. [Nursing-Care for the Elderly in 2015]. Tokyo: Ministry of Health, Labour and Welfare; 2003. Available from: http://www.mhlw.go.jp/topics/kaigo/kentou/15kourei/ index.html. Accessed August 3, 2012. Japanese.

6. Bellelli G, Frisoni GB, Bianchetti A, et al. Special care units for demented patients: a multicenter study. Gerontologist. 1998;38: 456-462.

7. Colombo M, Vitali S, Cairati M, Vaccaro R, Andreoni G, Guaita A. Behavioral and psychotic symptoms of dementia (BPSD) improvements in a special care unit: a factor analysis. Arch Gerontol Geriatr. 2007;1: $113-120$.

8. Mitsuyama H, Nohtomi A. An analysis of factors affecting outcome after discharge from a geriatric therapeutic unit. Rhonen Seishin Igaku Zasshi. 1999;10:1065-1073. Japanese [with English abstract].

9. Ukai K, Mizuno Y, Ozaki K, Sekiya T, Tomita K, Ito T. Physical complications of dementia: 12 months research in a special ward for the elderly with senile dementia. Psychogeriatrics. 2007;7:21-24.

10. Ukai K, Mizuno Y. Physical complications for elderly inpatients with senile dementia in the Imaise Branch of Ichinomiya City Hospital. Psychogeriatrics. 2009;9:167-172.

11. Ono T, Tamai A, Takeuchi D, et al. Predictors of length of stay in a ward for demented elderly: gender differences. Psychogeriatrics. 2010;10: 153-159.

12. Diagnostic and Statistical Manual of Mental Disorders - Text Revision (DSM-IV-TR). Washington, DC: American Psychiatric Association; 2000.

13. McKhann G, Drachman D, Folstein M, Katzman R, Price D, Stadlan EM. Clinical diagnosis of Alzheimer's disease: report of the NINCDSADRDA work group under the auspices of the Department of Health and Human Services Task Force on Alzheimer's disease. Neurology. 1984;34:939-944.

14. Román GC, Tatemichi TK, Erkinjuntti T, et al. Vascular dementia: diagnostic criteria for research studies. Report of the NINDS-AIREN international workshop. Neurology. 1993;43:250-260.

15. McKeith IG, Dickson DW, Lowe J, et al. Diagnosis and management of dementia with Lewy bodies: third report of the DLB Consortium. Neurology. 2005;65:1863-1872.

16. Neary D, Snowden JS, Gustafson L, et al. Frontotemporal lobar degeneration. A consensus on clinical diagnostic criteria. Neurology. 1998;51:1546-1554.

17. Hughes CP, Berg L, Danziger WL, Coben LA, Martin RL. A new clinical scale for the staging of dementia. Br J Psychiatr. 1982;140:566-572. 
18. Lawton MP, Brody EM. Assessment of older people: self-maintaining and instrumental activities of daily living. Gerontologist. 1969;9: 179-186.

19. Cummings JL, Mega M, Gray K, Rosenberg-Thompson S, Caruso DA, Hombeam J. The Neuropsychiatric Inventory: comprehensive assessment of psychopathology in dementia. Neurology. 1994;44:2308-2314.

20. Soto ME, Nourhashemi F, Arbus C, et al. Special acute care unit for older adults with Alzheimer's disease. Int J Geriatr Psychiatry. 2008;23: 215-219.

21. Ono T, Tamai A, Takeuchi D, Tamai Y. Predictors of outcomes from a ward for demented elderly: gender differences. Psychogeriatrics. 2010; 10:21-28.

22. US Food and Drug Administration. FDA public health advisory: deaths with antipsychotics in elderly patients with behavioral disturbances. Silver Spring, MD: US Food and Drug Administration. Available from: http://www.fda.gov/ForConsumers/ConsumerUpdates/ucm053171.htm. Accessed October 8, 2012

23. Wang PS, Schneeweiss S, Avorn J, et al. Risk of death in elderly users of conventional vs atypical antipsychotic medications. $N$ Engl J Med. 2005;353:2335-2341.
24. Yoshie S, Shiraishi H. A survey of wards for demented patients: demographics and correlations of hospitalization period. Rhonen Seishin Igaku Zasshi. 2007;2:197-207. Japanese [with English abstract].

25. Matsubara S. Psychiatric emergency service and physical complication service at a dementia-specialized ward. Rhonen Seishin Igaku Zasshi. 2007;9:1176-1183. Japanese.

26. Mitchell SL, Teno JM, Kiely DK, et al. The clinical course of advanced dementia. N Engl J Med. 2009;361:1529-1538.

27. Kitamura T, Kitamura M, Tanaka N, Kurata K. Gender difference in clinical features and care environment of the patients admitted to the dementia-specialized ward. Rhonen Seishin Igaku Zasshi. 2010;12: 1369-1376. Japanese [with English abstract].

28. Mizuno H. Measures and subjects about BPSD. Rhonen Seishin Igaku Zasshi. 2010;1:36-43. Japanese.

29. Matsubara S. The function of psychiatric hospitals in the treatment of dementia. Psychogeriatrics. 2012;12:127-130.
Neuropsychiatric Disease and Treatment

\section{Publish your work in this journal}

Neuropsychiatric Disease and Treatment is an international, peerreviewed journal of clinical therapeutics and pharmacology focusing on concise rapid reporting of clinical or pre-clinical studies on a range of neuropsychiatric and neurological disorders. This journal is indexed on PubMed Central, the 'PsycINFO' database and CAS.

\section{Dovepress}

The manuscript management system is completely online and includes a very quick and fair peer-review system, which is all easy to use. Visit http://www.dovepress.com/testimonials.php to read real quotes from published authors. 